Pacific Journal of Mathematics

INFLATION AND DEFLATION FOR ALL DIMENSIONS 


\section{INFLATION AND DEFLATION FOR ALL DIMENSIONS}

\section{ERNST SNAPPER}

We assume that a finite group $G$ acts on the left on finite sets $X$ and $Y$, and that there is given a function $f: X \rightarrow Y$. We assume that $f(\sigma x)=\sigma f(x)$ for all $\sigma \in G$ and $x \in X$; and that $f^{-1}(y)$ has the same number $h$ of elements for all $y \in Y$. We show that the cohomology groups $H^{r}(X ; G, A)$ and $H^{r}(Y ; \mathbf{G}, A)$ of the permutation representations $(G, X)$ and $(G, Y)$ with values in a $G$-module $A$ are interrelated by homomorphisrns inflation $_{r}: \quad H^{r}(Y ; G, A) \rightarrow H^{r}(X ; G, A)$ and deflation $_{r}$ : $H^{r}(Y ; G, A) \rightarrow H^{r}(Y ; G, A)$, for all $r \in Z$. The main properties of $\inf _{r}\left(\right.$ inflation $\left._{r}\right)$ and $\operatorname{def}_{r}\left(\right.$ deflation $\left._{r}\right)$ are:

I. For all $r \in Z$, $\operatorname{def}_{r}$ inf $_{r}: H^{r}(Y ; G, A) \rightarrow H^{r}(Y ; G, A)$ consists of multiplying the elements of $H^{r}(Y ; G, A)$ by $h^{q}$, where $q \geqq 1$ and $q$ depends on $r$.

II. If for some $r \in Z, H^{r}(Y$; $G, A)$ is uniquely divisible by $h$, inf ${ }_{r}$ is a monomorphism and $\operatorname{def}_{r}$ is an epimorphism and $H^{r}(X ; G, A)=\operatorname{im}\left(\inf _{r}\right) \oplus \operatorname{ker}\left(\operatorname{def}_{r}\right)$, where $\oplus$ denotes the direct sum of abelian groups.

III. $H^{r}(Y ; G, A)$ is uniquely divisible by $h$ for all $r \in Z$ in each of the following two cases.

IIIa. $A$ is uniquely divisible by $h$.

IIIb. $(h, m)=1$ where $m$ is the index of $(G, Y)$.

We then study the special case where the permutation representations $(G, X)$ and $(G, Y)$ are transitive and where $(G, X)$ is furthermore free of fixed points. Since the classical inflation and deflation mappings fall under this heading, we have now extended these mappings to all of $Z$. We describe the six mappings inf $f_{r}$ and $\operatorname{def}_{r}$ for $r=0, \pm 1$ explicity in terms of trace mappings, augmentation ideals and crossed homomorphisms.

$G$ stands for a finite group. For every normal subgroup $H$ of $G$ and $G$-module $A$, the inflation (or lift) mapping $H^{r}\left(G / H, A^{H}\right) \rightarrow H^{r}(G$, $A$ ) is well known for $r \geqq 1 ; A^{H}$ always denotes the submodule of $A$ whose elements are left fixed by $H$. Dually, there is available the deflation mapping $H^{r}(G, A) \rightarrow H^{r}\left(G / H, A^{H}\right)$ for $r \leqq-2$ (see [7]). In the present paper we extend the inflation and deflation mappings to all $r \in Z$. ( $Z$ denotes the ring of the rational integers.) We develop the theory for arbitrary permutation representations (see [6] for the cohomology of permutation representations) which includes the case

Received March 5, 1964. This research was supported in part by the National Science Foundation grant NSF-GP722. 
that $H$ is not normal.

The fact that the inflation mapping followed by the deflation mapping consists of multiplying by a power of [H:1] (see Theorem 5.1 ), indicates that these mappings behave particularly nicely if $A$ is uniquely divisible by [H:1], or if $H$ is a Hall subgroup of $G$. These cases are worked out in $\S 6,7,8,11,12$ and 13 and are needed for the author's forthcoming paper on duality in the cohomology of permutation representations. The study of deflation in dimension 1 brings to the fore natural endomorphisms of the group of crossed homomorphisms from $G$ to $A$. There is one such endomorphism for each subgroug of $G$. (see $\S 15$ and 16.)

1. Inflation for chains. $X$ stands for a finite set and $(G, X)$ for a permutation representation (see the introduction of [6]); i.e., $\sigma x \in X$ for all $x \in X$ and $\sigma \in G$, and $(\sigma \tau) x=\sigma(\tau x)$ and $1 x=x$ for all $\sigma, \tau \in G ; 1$ always denotes the unit element of the group under discussion. Let $(L, Y)$ be a second permutation representation of some finite group $L$ acting on some finite set $Y$, and let $\theta=(\varphi, f):(G, X) \rightarrow(L$, $Y$ ) be a morphism of permutation representations (see the introduction of [6]); i.e., $\varphi: G \rightarrow L$ is a group homomorphism and $f: X \rightarrow Y$ is a function where $f(\sigma x)=\varphi(\sigma) f(x)$ for all $\sigma \in G$ and $x \in X$. The $r$ th chain group $C_{r}(X ; G)$ of the standard complex $C .(X ; G)$ of $(G, X)$ is the $G$ module $Z\left[X^{q}\right]$, where $X^{q}$ is the cartesian product of $X$ with itself $q$ times; $q=r+1$ if $r \geqq 0$ and $q=-r$ if $r<0$ (see $\S 1$ of [6]; the same definitions hold of course for $(L, Y)$.) The function $\left(x_{1}, \cdots x_{q}\right) \rightarrow$ $\left(f\left(x_{1}\right), \cdots, f\left(x_{q}\right)\right)$ from $X^{q}$ to $Y^{q}$ can be extended by linearity to a homomorphism $\alpha_{r}: Z\left[X^{q}\right] \rightarrow Z\left[Y^{q}\right]$ which is a $G$-homomorphism if we regard the $L$-module $Z\left[Y^{q}\right]$ as a $G$-module under $\varphi: G \rightarrow L$. All this gives rise to the diagram:

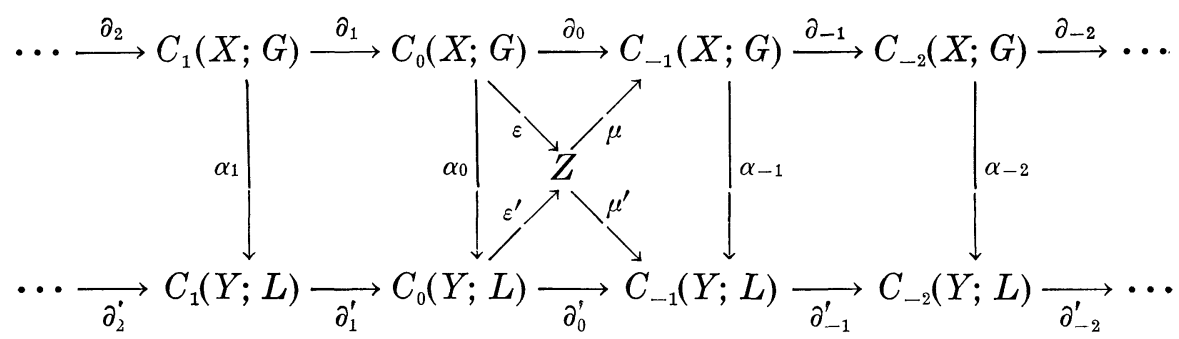

We have primed the differentiation mappings $\partial_{r}^{\prime}$ and augmentation mappings $\varepsilon^{\prime}, \mu^{\prime}$ of the complex $C .(Y ; L)$. We know from $\S 1$ of [6] that $\mu \varepsilon=\partial_{0}$ and that $\mu^{\prime} \varepsilon^{\prime}=\partial_{0}^{\prime}$; and $\S 13$ of [6] tells us that $\partial_{r}^{\prime} \alpha_{r}=$ $\alpha_{r-1} \partial_{r}$ for $r \geqq 1$ and that $\varepsilon^{\prime} \alpha_{0}=\varepsilon$. The reason why one shies away from studying $\alpha_{r}$ for $r<0$ is that these commutativity relations fail 
for $r<0$. We show however that they fail by so little that these maps $\alpha_{r}$ are still very useful for $r<0$.

We assume for the remainder of this paper that $f^{-1}(y)$ contains the same number of elements for all $y \in Y$, and denote this number by $h$. This implies of course that $f: X \rightarrow Y$ is an epimorphism and hence that $\alpha_{r}$, for all $r \in Z$, is an epi. Conversely, if $f$ is an epi and the permutation representation $(G, X)$ is transitive, the number of elements in $f^{-1}(y)$ does not depend on $y$. This follows easily from the fact that for every morphism of permutation representations the partitioning $X=\bigcup f^{-1}(y)$ of $X$ consists of domains of imprimitivity of ( $G$, $X)$. (See $\$ 146$ of [2] for domains of imprimitivity.)

We replace the differentiation operator $\partial_{r}^{\prime}$ of $C .(Y ; L)$ by $h \partial_{r}^{\prime}$ if $r<0$, but leave $\partial_{r}^{\prime}$ unchanged for $r<0$. We also change $\mu^{\prime}$ to $h \mu^{\prime}$ but leave $\varepsilon^{\prime}$ unchanged. We now show that the following diagram displays a chain mapping of complexes.

(I)

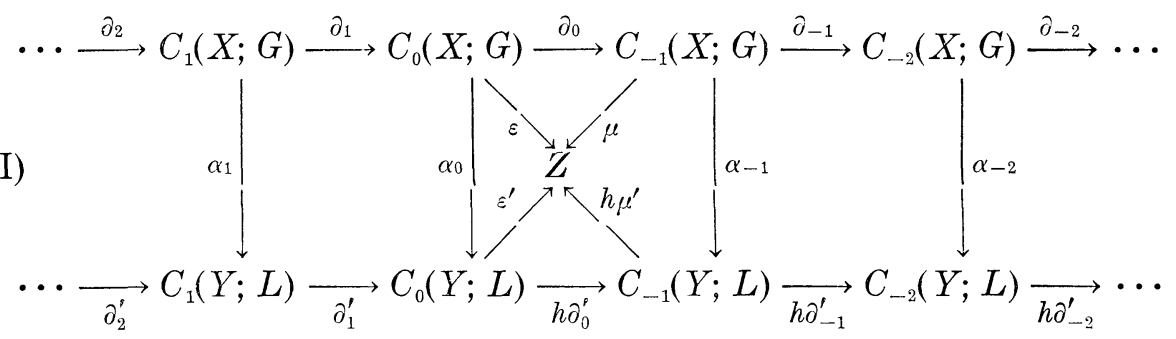

Proposition 1.1. The upper row of diagram (I) is a $G$-complex and the lower row is an $L$-complex. The diagram is completely commutative, that is;

(1) $\partial_{r}^{\prime} \alpha_{r}=\alpha_{r-1} \partial_{r}$ for $r \geqq 1$;

(2) $\varepsilon^{\prime} \alpha_{0}=\varepsilon$;

(3) $\mu \varepsilon=\partial_{0}$;

(4) $h \mu^{\prime} \varepsilon^{\prime}=h \partial_{0}^{\prime}$;

(5) $\alpha_{-1} \mu=h \mu^{\prime}$;

(6) $h \partial_{r}^{\prime} \alpha_{r}=\alpha_{r-1} \partial_{r}$ for $r \leqq-1$.

The chain mapping $\left\{\alpha_{r}, r \in Z\right\}$ is an epimorphism and a $G$-mapping if we consider the lower row as a $G$-complex under $\varphi: G \rightarrow L$.

Proof. The upper row is the $G$-complex $C .(X ; G)$. The fact that $C .(Y ; L)$ is an $L$-complex implies immediately that the lower row is also an $L$-complex. The first three commutativity relations have been discussed above and (4) follows from $\mu^{\prime} \varepsilon^{\prime}=\partial_{0}^{\prime}$. For (5) we observe that $\alpha_{-1} \mu(1)=\alpha_{-1} \Sigma_{x \in X} x=\Sigma_{x \in X} f(x)=h \Sigma_{y \in Y} y=h \mu^{\prime}(1)$. For $\quad$ (6) we select $\left(x_{1}, \cdots, x_{r}\right) \in X^{r}$ and use the definition of $\partial_{-r}$ of $\S 1$ of [6] to 
compute that $\alpha_{--r-1} \partial_{-r r}\left(x_{1}, \cdots, x_{r}\right)=\alpha_{-r-1}\left(\Sigma_{x \in X}\left(x, x_{1}, \cdots, x_{r}\right)+\sum_{i=1}^{r}(-1)^{i}\right.$ $\left.\Sigma_{x \in X}\left(x_{1}, \cdots, x_{i}, x, x_{i+}, \cdots x_{r}\right)\right)=\sum_{x \in X}\left(f(x), f\left(x_{1}\right), \cdots, f\left(x_{r}\right)\right)+\sum_{i-1}^{r}(-1)^{i}$ $\Sigma_{x \in X}\left(f\left(x_{1}\right), \cdots, f\left(x_{i}\right), f(x) f\left(x_{i+1}\right), \cdots, f\left(x_{r}\right)\right)=h \Sigma_{y \in Y}\left(y, f\left(x_{1}\right), \cdots, f\left(x_{r}\right)\right)+$ $+h \Sigma_{i=1}^{r}(-1)^{i} \Sigma_{y \in Y}\left(f\left(x_{1}\right), \cdots, f\left(x_{i}\right), y, f\left(x_{i+1}\right), \cdots, f\left(x_{r}\right)\right)=h \partial_{-r}^{\prime}\left(f\left(x_{1}\right), \cdots\right.$, $\left.f\left(x_{r}\right)\right)=h \partial_{-r}^{\prime} \alpha_{-r}\left(x_{1}, \cdots, x_{r}\right)$. Finally, the fact that $\alpha_{r}: C_{r}(X ; G) \rightarrow C_{r}(Y$; $L)$ is an epimorphism and may be regarded as a $G$-homomorphism has been mentioned previously. Done.

One should be careful to observe that the lower row of diagram (I) may not be acyclic any longer. True, its $r$ th cycle group is the same as the $r$ th cycle group of the acyclic complex $C .(Y ; L)$, because $C_{r}(Y ; L)=Z\left[Y^{q}\right]$ is without torsion for all $r \in Z$. However, if $r \leqq-1$, the $r$ th boundary group of the lower row of diagram (I) is $h B_{r}$ where $B_{r}$ denotes the $r$ th boundary group of $C .(Y ; L)$.

It is convenient to think of the mappings $\alpha_{r}$ as the "inflation mappings for chains" because, if $r \geqq 1, \alpha_{r}$ gives rise to the customary inflation mapping (see Definition 4.1). If however $r \leqq 0$, either $\alpha_{r}$ or $h \alpha_{r}$ is used to define the inflation mapping (same definition).

2. Deflation for chains. We define, for every $r \in Z$, a homomorphism $\beta_{r}: C_{r}(Y ; L) \rightarrow C_{r}(X ; G)$. Again, $C_{r}(Y ; L)=Z\left[Y^{q}\right]$, where $q=r+1$ if $r \geqq 0$ and $q=-r$ if $r<0$. The mapping $\left(y_{1}, \cdots, y_{q}\right) \rightarrow$ $\Sigma\left(x_{i_{1}}, \cdots, x_{i_{q}}\right)$, where the summation is over all $q$-tuples of the cartesian product $f^{-1}\left(y_{1}\right) \times \cdots \times f^{-1}\left(y_{q}\right)$, maps the $Z$-base of $Z\left[Y^{q}\right]$ into $Z\left[X^{q}\right]=C_{r}(X ; G)$. We define $\beta_{r}$ as the extension by linearity of this mapping to $Z\left[Y^{q}\right]$. We observe that $\beta_{r}$ is the dual of the mapping $\alpha_{-r-1}$ in the following sense. $C_{r}(Y ; L)$ may be regarded as $\operatorname{Hom}_{z}\left(C_{-r-1}\right.$ $(Y ; L), Z)$ and similarly, for $C_{r}(X ; G)$. (See $\S 1$ of [6].) If we apply the functor $\left.\operatorname{Hom}_{Z}{ }^{*}, Z\right)$ to the homomorphism $\alpha_{-r-1}: C_{-r-1}(X ; G) \rightarrow$ $C_{-r-1}(Y ; L)$ we obtain the homomorphism $\beta_{r}: C_{r}(Y ; L) \rightarrow C_{r}(X ; G)$. This observation makes the following proposition into an easy corollary of Proposition 1.1.

Proposition 2.1. The upper row of diagram (II) (see below) is a $G$-complex and the lower row is an $L$-complex. The diagram is completely commutative, that is:

(1) $\partial_{r} \beta_{r}=\beta_{r-1} h \partial_{r}^{\prime}$ for $r \geqq 1$;

(2) $\varepsilon \beta_{0}=h \varepsilon^{\prime}$;

(3) $\mu \varepsilon=\partial_{0}$;

(4) $\mu^{\prime} h \varepsilon^{\prime}=h \partial_{0}^{\prime}$

(5) $\beta_{-1} \mu^{\prime}=\mu$;

(6) $\partial_{r} \beta_{r}=\beta_{r-1} \partial_{r}^{\prime}$ for $r \leqq-1$.

The chain mapping $\left\{\beta_{r} ; r \in Z\right\}$ is a monomorphism and is a $G$-mapping if we consider the lower row as a $G$-complex under $\varphi: G \rightarrow L$. 


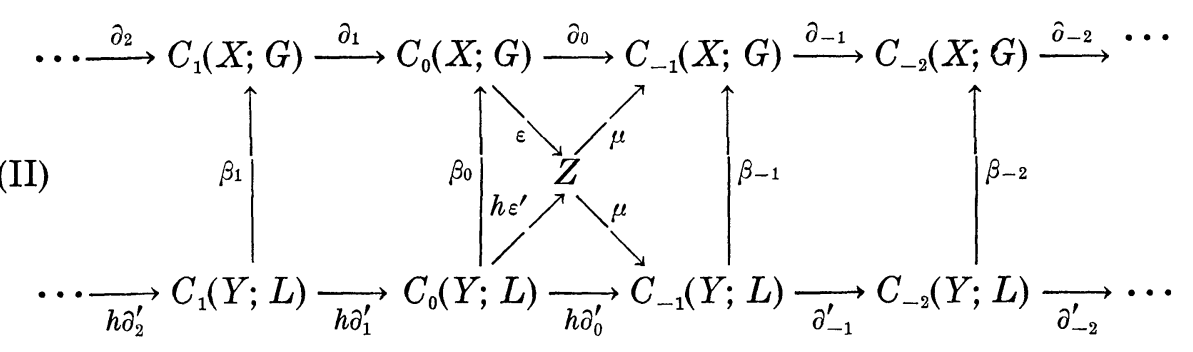

Observe that the lower rows of diagrams (I) and (II) are not the same but correspond to one another under the functor $\operatorname{Hom}_{z}\left({ }^{*}, Z\right)$. It is convenient to think of the mappings $\beta_{r}$ as the "deflation mappings for chains" because, if $r \leqq-2, \beta_{r}$ gives rise to the deflation mapping defined in [7]. If however $r \geqq-1$, either $\beta_{r}$ or $h \beta_{r}$ is used to define the deflation mapping (see Definition 5.1).

PRoposition 2.2. $\alpha_{r} \beta_{r}-h^{r+1}$ if $r \geqq 0$ and $\alpha_{r} \beta_{r}=h^{-r}$ if $r \leqq-1$. Here, $h^{q}$ denotes the endomorphism of $C_{r}(Y ; L)$ which consists of multiplying its elements by $h^{q}$.

Proof. The $Z$-base of $C_{r}(Y ; L)$ consists of the $q$-tuples $\left(y_{1}, \cdots\right.$, $\left.y_{q}\right) \in Y^{q}$. Furthermore, $\alpha_{r} \beta_{r}\left(y_{1}, \cdots, y_{q}\right)=\alpha_{r} \Sigma\left(x_{i_{1}}, \cdots, x_{i_{q}}\right)=\Sigma\left(f\left(x_{i_{1}}\right), \cdots\right.$, $f\left(x_{i_{q}}\right)$ ) where the summation is over the $h^{q} q$-tuples $\left(x_{i_{1}}, \cdots, x_{i_{q}}\right)$ of the cartesian product $f^{-1}\left(y_{1}\right) \times \cdots \times f^{-1}\left(y_{q}\right)$. Hence the last sum is equal to $h^{q}\left(y_{1}, \cdots, y_{q}\right)$. Done.

3. Inflation and deflation for cochains. We now have to "hom" diagrams (I) and (II) with modules. Although it is possible to work simultaneously with a $G$-module and an $L$-module, we restrict ourselves to the case which is of principal interest for group theory. We assume for the remainder of this paper that $G=L$ and that $\varphi$ is the identity mapping of $G$. Furthermore, $A$ stands for a G-module.

If we apply the functor $\left.\operatorname{Hom}_{G}{ }^{*}, A\right)$ to the chain complex $C .(X$; $G$ ), we obtain the cochain complex $\mathrm{C}^{\cdot}(X ; G, A)$ (see $\S 2$ of [6]). We denote the $r$ th cochain group of $C^{\cdot}(X ; G, A)$ by $C^{r}(X ; G, A)$ and treat the permutation representation $(G, Y)$ in the same way. Hence, under the functor $\left.\operatorname{Hom}_{G}{ }^{*}, A\right)$, the mappings $\alpha_{r}: C_{r}(X ; G) \rightarrow C_{r}(Y ; G)$ and $\beta_{r}: C_{r}(Y ; G) \rightarrow C_{r}(X ; G)$ become, respectively, mappings $a_{r}: C^{r}(Y$; $G, A) \rightarrow C^{r}(X ; G, A) \quad$ and $\quad b_{r}: C^{r}(X ; G, A) \rightarrow C^{r}(Y ; G, A) ; \quad$ here, $a_{r}=$ $\operatorname{Hom}_{\theta}\left(\alpha_{r}, 1_{A}\right)$ and $b_{r}=\operatorname{Hom}_{\theta}\left(\beta_{r}, 1_{A}\right)$ where $1_{A}$ denotes the identity of $A$. When we apply the same functor to diagrams (I) and (II) we obtain, respectively, diagrams (III) and (IV); and Propositions 1.1, 2.1 and 2.2 give Proposition 3.1. 
$\cdots \stackrel{\delta_{-3}}{\longrightarrow} C^{-2}(X ; G, A) \stackrel{\delta_{-2}}{\longrightarrow} C^{-1}(X ; G, A) \stackrel{\delta^{-1}}{\longrightarrow} C^{0}(X ; G, A) \stackrel{\delta_{0}}{\longrightarrow} C^{1}(X ; G, A) \stackrel{\delta_{1}}{\longrightarrow} \cdots$

(III)
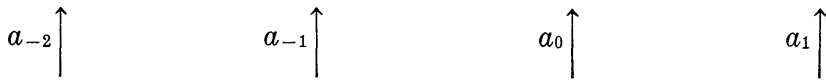

$\cdots \underset{h \delta_{-3}^{\prime}}{\longrightarrow} C^{-2}(Y ; G, A) \underset{h \delta_{-2}^{\prime}}{\longrightarrow} C^{-1}(Y ; G, A) \underset{h \delta_{-1}^{\prime}}{\longrightarrow} C^{0}(Y ; G, A) \underset{\delta_{0}^{\prime}}{\longrightarrow} C^{1}(Y ; G, A) \underset{\delta_{1}^{\prime}}{\longrightarrow} \cdots$

$\cdots \stackrel{\delta_{-3}}{\longrightarrow} C^{-2}(X ; G, A) \stackrel{\delta_{-2}}{\longrightarrow} C^{-1}(X, G, A) \stackrel{\delta_{-1}}{\longrightarrow} C^{0}(X ; G, A) \stackrel{\delta_{0}}{\longrightarrow} C^{1}(X ; G, A) \stackrel{\delta_{1}}{\longrightarrow} \cdots$

(IV)
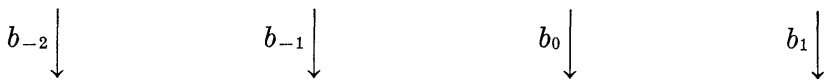

$\cdots \underset{\delta_{-3}^{\prime}}{\longrightarrow} C^{-2}(Y ; G, A) \underset{\delta_{-2}^{\prime}}{\longrightarrow} C^{-1}(Y ; G, A) \underset{h \delta_{-1}^{\prime}}{\longrightarrow} C^{0}(Y ; G, A) \underset{h \delta_{0}^{\prime}}{\longrightarrow} C^{1}(Y, G, A) \underset{h \delta_{1}^{\prime}}{\longrightarrow} \cdots$

Proposition 3.1. All four rows in diagrams (III) and (IV) are complexes of abelian groups, and both diagrams are commutative diagrams. The chain mapping $\left\{a_{r} ; r \in Z\right\}$ is a monomorphism, but the chain mapping $\left\{b_{r} ; r \in Z\right\}$ is not necessarily an epimorphism. Furthermore, $b_{r} a_{r}=h^{r+1}$ if $r \geqq 0$ and $b_{r} a_{r}=h^{-r}$ if $r \leqq-1$; here, $h^{q}$ denotes the endomorphism of $C^{r}(Y ; G, A)$ which consists of multiplying its elements by $h^{q}$.

It is clear from the previous sections that it is convenient to think of the mappings $a_{r}$ and $b_{r}$ as, respectively, the "inflation mapping" and "deflation mapping" for cochains.

4. Inflation for cohomology groups. We denote, as in [6], the $r$ th cocycle group (coboundary group, cohomology group) of the complex $C^{\cdot}(X ; G, A)$ by $Z^{r}(X ; G, A),\left(B^{r}(X ; G, A), H^{r}(X, G, A)\right)$; we do of course the same for $C \cdot(Y ; G, A)$. We read immediately from diagram (III) that $a_{r}\left(Z^{r}(Y ; G, A)\right) \subset Z^{r}(X ; G, A)$ for all $r \in Z$; and that $a_{r}\left(B^{r}(Y ; G, A)\right) \subset B^{r}(X ; G, A)$ if $r \geqq 1$. If $r \leqq 0, a_{r}$ may not transform coboundaries into coboundaries (see Example 9.1); this depends on the nature of our morphism $(G, X) \rightarrow(G, Y)$ and the $G$-module $A$. However, diagram (III) does tell us immediately that $h a_{r}\left(B^{r}(Y ; G, A)\right) \subset$ $B^{r}(X ; G, A)$ and that $h a_{r}\left(Z^{r}(Y ; G, A)\right) \subset Z^{r}(X ; G, A)$ for all $r \in Z$.

The above implies the following for the cohomology groups. The homomorphism $h a_{r}$ always induces a homomorphism $\left(h a_{r}\right)^{*}: H^{r}(Y ; G$, $A) \rightarrow H^{r}(X ; G, A)$ for all $r \in Z$. The homomorphism $a_{r}$ induces a homomorphism $a_{r}^{*}: H^{r}(Y ; G, A) \rightarrow H^{r}(X ; G, A)$ for $r \geqq 1$ but, depending on the morphism $(G, X) \rightarrow(G, Y)$ and on $A$, not for $r \leqq 0$. Whenever $a_{r}^{*}$ exists, that's the mapping we want. If however $a_{r}^{*}$ does not exist we should not despair but be satisfied with $\left(h a_{r}\right)^{*}$. The following definition reflects this attitude.

Definition 4.1. Let $r \in Z$. If it happens that $a_{r}\left(B^{r}(Y ; G, A)\right) \subset$ 
$B^{r}(X ; G, A)$, we call the homomorphism $a_{r}^{*}: H^{r}(Y ; G, \mathrm{~A}) \rightarrow H^{r}(X ; G, A)$ the inflation mapping or lift mapping for dimension $r$. If $a_{r}\left(B^{r}(Y\right.$; $G, A)) \not \subset B^{r}(X ; G, A)$, we call the homomorphism $\left(h a_{r}\right)^{*}: H^{r}(Y ; G, A) \rightarrow$ $H^{r}(X ; G, A)$ the inflation mapping or lift mapping. We denote the inflation mapping by inf or $\inf _{r}$.

The above definition gives the customary inflation mapping when $r \geqq 1$. We repeat that, when $r \leqq 0$, it depends on the morphism $(G, X) \rightarrow(G, Y)$ and the module $A$ whether $\inf _{r}=a_{r}^{*}$ or $\inf _{r}=\left(h a_{r}\right)^{*}$.

REMARK 4.1. One could obviously have proceeded differently. Namely, diagram (III) shows that $a_{r}$ always induces a homomorphism from the $r$ th cohomology group $H^{r}$ of the lower row of that diagram into $H^{r}(X ; G, A)$. The groups $H^{r}$ for $r \leqq 0$ seem to be of no particular interest for group theory which is why we proceeded as in Definition 4.1.

EXAMPLE 4.1. Consider the morphism of permutation representations $\left(1_{G}, f\right):(G, G) \rightarrow(G, G / H)$. Here, $X=G$ and the permutation representation $(G, G)$ consists of $G$ acting by left multiplication on itself. Furthermore $H$ is a subgroup of $G$, not necessarily normal, and $Y$ is the set $G / H$ of the left cosets of $H$. The permutation representation $(G, G / H)$ consists of $G$ acting on these cosets by left multiplication. Finally, $f(\sigma)=\sigma H$ for $\sigma \in G$. The number of elements in $f^{-1}(\sigma H)$ is the order $h$ of $H$ and hence is independent of $\sigma H$. Consequently, Definition 4.1 applies and $\inf _{r}: H^{r}(G / H ; G, A) \rightarrow H^{r}(G ; G, A)$ is defined for all $r \in Z$. As is well known, $H^{r}(G ; G, A)$ is the classical cohomology group $H^{r}(G, A)$, and $H^{r}(G / H ; G, A)$ is the relative group $H^{r}(G: H, A)$ defined in [1]. If $r \geqq 1, \inf _{r}$ coincides with the inflation mapping defined in $\S 7$ of [1]. If $H$ is a normal subgroup of $G, H^{r}(G: H, A)$ is isomorphic with the classical cohomology group $H^{r}\left(G / H, A^{H}\right)$ (see the Corollary on page 68 of [1]) and we obtain, if $r \geqq 1$, the customary inflation mapping from $H^{r}\left(G / H, A^{H}\right)$ into $H^{r}(G$, $A)$. We shall frequently come back to this example.

5. Deflation for cohomology groups. We read from diagram (IV) that $b_{r}\left(B^{r}(X ; G, A)\right) \subset B^{r}(Y ; G, A)$ for all $r \in Z$; and that $b_{r}\left(Z^{r}(X\right.$; $G, A)) \subset Z^{r}(Y ; G, A)$ if $r \leqq-2$. If $r \geqq-1, b_{r}$ may not transform cocycles into cocycles. Diagram (IV) also tells us that $h b_{r}\left(Z^{r}(X ; G\right.$, $A)) \subset Z^{r}(Y ; G, A)$ and that $h b_{r}\left(B^{r}(X ; G, A)\right) \subset B^{r}(Y ; G, A)$ for all $r \in Z$.

Consequently, $h b_{r}$ induces a homomorphism $\left(h b_{r}\right)^{*}: H^{r}(X ; G, A) \rightarrow$ $H^{r}(Y: G, A)$ for all $r \in Z$. The homomorphism $b_{r}$ induces a homomorphism $b_{r}^{*} ; H^{r}(X ; G, A) \rightarrow H^{r}(Y ; G, A)$ for $r \leqq-2$ but, depending on the morphism $(G, X) \rightarrow(L, Y)$ and the module $A$, not for $r \leqq-1$. 
We proceed as in the case of inflation.

Definition 5.1. Let $r \in Z$. If it happens that $b_{r}\left(Z^{r}(X ; G, A)\right) \subset$ $Z^{r}(Y ; G, A)$, we call the homomorphism $b_{r}^{*}: H^{r}(X ; G, A) \rightarrow H^{r}(Y ; G, A)$ the deflation mapping for dimension $r$. If $b_{r}\left(Z^{r}(X ; G, A)\right) \not \subset Z^{r}(Y ; G, A)$, we call the homomorphism $\left(h b_{r}\right)^{*}: H^{r}(X ; G, A) \rightarrow H^{r}(Y ; G, A)$ the deflation mapping. We denote the deflation mapping by def or $\operatorname{def}_{r}$.

We repeat that, when $r \leqq-2, \operatorname{def}_{r}=\left(b_{r}\right)^{*}$. If $r \geqq-1$, it depends on the morphism $(G, X) \rightarrow(G, Y)$ and the $G$-module $A$ whether $\operatorname{def}_{r}=b_{r}^{*}$ or $\operatorname{def}_{r}=\left(h b_{r}\right)^{*}$. Remark 4.1 applies of course equally well to deflation.

EXAMPle 5.1. Consider the morphism $\left(1_{G}, f\right):(G, G) \rightarrow(G, G / H)$ of Example 4.1. Definition 5.1 defines the deflation mapping def ${ }_{r}$ : $H^{r}(G, A) \rightarrow H^{r}(G: H, A)$ for all $r \in Z$. If $H$ is a normal subgroup of $G$, $\operatorname{def}_{r}$ maps $H^{r}(G, A)$ into $H^{r}\left(G / H, A^{H}\right)$; if furthermore $r \leqq-2$, $\operatorname{def}_{r}$ coincides with the deflation mapping studied in [7].

THEOREM 5.1. Let $h^{q}$ denote the endomorphism of $H^{r}(Y ; G, A)$ which consists of multiplying its elements by $h^{q}$. For each $r \in Z$ there exists an integer $q \geqq 1$, depending on $r$, such that def $f_{r}$ inf $f_{r}=$ $h^{q}$.

Proof. $\operatorname{def}_{r} \inf _{r}$ is equal to $b_{r}^{*} a_{r}^{*}$ or to $\left(h b_{r}\right)^{*} a_{r}^{*}$ or $b_{r}^{*}\left(h a_{r}\right)^{*}$ or $\left(h b_{r}\right)^{*}\left(h a_{r}\right)^{*}$. Proposition 3.1 tells us that $b_{r} a_{r},\left(h b_{r}\right) a_{r}, b_{r}\left(h a_{r}\right)$ and $\left(h b_{r}\right)$ $\left(h a_{r}\right)$ all consist of multiplying the elements of $C^{r}(Y ; G, A)$ by a positive power of $h$. Done.

We now study various special instances of inflation and deflation. Hereto, we need some material on uniquely divisible modules.

6. Uniquely divisible modules. In this whole section, $k \in Z$ stands for a fixed, nonzero integer. If $F$ is a module (i.e., an abelian group written additively) we denote the identity mapping of $F$ onto itself by $1_{F}$. Hence, $k 1_{F}$ denotes the endomorphism of $F$ which consists of multiplying its elements by $k$. As always, $F$ is called divisible by $k$ if $k 1_{F}$ is an epimorphism; and $F$ is called uniquely divisible by $k$ if $k 1_{F}$ is an automorphism.

Proposition 6.1. Let $0 \longrightarrow D \stackrel{i}{\longrightarrow} E \stackrel{j}{\longrightarrow} F \longrightarrow 0$ be an exact sequence of modules. If two of them are uniquely divisible by $k$, so is the third.

Proof. Consider the commutative diagram 


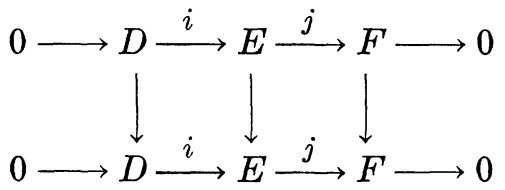

where the vertical arrows denote, respectively, $k 1_{D}, k 1_{E}$ and $k 1_{F}$. We conclude from the "5 lemma" (see [3], page 5) that, if two of the vertical arrows are automorphisms, so is the third. Done.

Proposition 6.2. Let $v: E \rightarrow F$ be a homomorphism from the module $E$ to the module $F$. If $E$ and $F$ are both uniquely divisible by $k$, so are $\operatorname{ker}(v), \operatorname{coker}(v), \operatorname{im}(v)$ and $\operatorname{coim}(v)$. (Coim stands for coimage.)

Proof. Since $E$ is divisible by $k, \operatorname{im}(v)$ is evidently divisible by $k$. The fact that, actually, $\operatorname{im}(v)$ is uniquely divisible by $k$ then follows from the fact that $k 1_{F}$ is a monomorphism. This also takes care of $\operatorname{coim}(v) \simeq \operatorname{im}(v)$. We now apply Proposition 6.1 to the exact sequences $0 \rightarrow \operatorname{im}(v) \rightarrow F \rightarrow \operatorname{coker}(v) \rightarrow 0$ and $0 \rightarrow \operatorname{ker}(v) \rightarrow E \rightarrow \operatorname{coim}(v) \rightarrow$ 0 and we are done.

REMARKS 6.1. Propositions 6.1 and 6.2 together say that the category of modules which are uniquely divisible by $k$ is a complete subcategory of the category of abelian groups (see page 138 of [5]). This subcategory is not "épaisse" (same reference) since the additive group of $Z$ is a subgroup of the additive group of the rational numbers; the latter group is uniquely divisible by $k$ but, if $k \neq \pm 1$, the first one is not.

Proposition 6.3. Let $E$ and $F$ be two $A$-modules where $\Lambda$ is some ring with unit element. If one of the modules is uniquely divisible by $k$, so is $\operatorname{Hom}_{4}(E, F)$.

Proof. Suppose that $k 1_{E}$ is an automorphism. Then, $\operatorname{Hom}_{A}\left(k 1_{E}\right.$, $\left.1_{F}\right): \operatorname{Hom}_{4}(E, F) \rightarrow \operatorname{Hom}_{4}(E, F)$ is an automorphism, and it consists of course of multiplying the elements of $\operatorname{Hom}_{A}(E, F)$ by $k$. We proceed similarly if $k 1_{F}$ is an automorphism. Done.

We now return to our permutation representation $(G, X)$. Since $(G, X)$ is entirely arbitrary, Lemma 6.1 is valid for all permutation representations.

LEMmA 6.1. Let $A$ be a G-module which is uniquely divisible by k. Then, $H^{r}(X ; G, A)$ is uniquely divisible by $k$ for all $r \in Z$. 
Proof. Let $r \in Z . \quad C^{r}(X ; G, A)=\operatorname{Hom}_{G}\left(C_{r}(X ; G), A\right)$ is uniquely divisible by $k$ by Proposition 6.3. We apply Proposition 6.2 to the homomorphisms $C^{r-1}(X, G, A) \rightarrow C^{r}(X ; G, A) \rightarrow C^{r+1}(X ; G, A)$ and find that the cocycle group $Z^{r}(X ; G, A)$ and the coboundary group $B^{r}(X$, $G, A)$ are uniquely divisible by $k$. Since $H^{r}(X ; G, A)$ is the cokernel of the inclusion mapping $B^{r}(X ; G, A) \rightarrow Z^{r}(X ; G, A)$, the same proposition gives the desired result.

REMARK 6.2. Lemma 6.1 gives a cute proof of the well known fact that $H^{r}(G, A)=0$ if $A$ is uniquely divisible by the order $n$ of $G$. Namely, $n H^{r}=0$ and, by Lemma 6.1, $n H^{r}(G, A)=H^{r}(G, A)$. More generally, if $A$ is uniquely divisible by the index of the permutation representation $(G, X)$, then $H^{r}(X ; G, A)=0$ for all $r \in Z$. (See Corollary 10.2 of [6].)

7. The case that $H^{r}(Y ; G, A)$ is uniquely divisible by $h$. We recall that the set $Y$ is partitioned into the domains of transitivity $T_{1}, \cdots, T_{u}$ of the permutation representation $(G, Y)$. If $T_{i}$ has $m_{i}$ elements, the greatest common divisor $m$ of $m_{1}, \cdots, m_{u}$ is called the index of $(G, Y)$ (see $\S 4$ of [6]).

Lemma 7.1. Let $d=(h, m)$. If $A$ is uniquely divisible by $d$, then $H^{r}(Y ; G, A)$ is uniquely divisible by $h$ for all $r \in Z$.

Proof. $H^{r}(Y ; G, A)$ is uniquely divisible by $d$ by Lemma 6.1 , and $m H^{r}(Y ; G, A)=0$ by Corollary 10.2 of [6]. Done.

The following proposition is an immediate corollary of Lemma 7.1.

Proposition 7.1. In each of the following two cases $H^{r}(Y, G, A)$ is uniquely divisible by $h$ for all $r \in Z$.

(a) $A$ is uniquely divisible by $h$.

(b) $(h, m)=1$.

EXAmple 7.1. Case $(b)$ of Proposition 7.1 is important for Hall subgroups. ( $A$ subgroup $H$ of $G$ is called a Hall subgroup if the order of $H$ is relatively prime to the index $[G: H]$ of $H$.) In the morphism $(G, G) \rightarrow(G, G / H)$ of Example 4.1 , the index of $(G, G / H)$ is the index $[G: H]$; hence, $(h, m)=1$ if and only if $H$ is a Hall subgroup of $G$.

THEOREM 7.1. Let $r \in Z$ and let $H^{r}(Y ; G, A)$ be uniquely divisible ty h. Then, inf $f_{r}$ is a monomorphism and def $f_{r}$ is an epimorphism; and $H^{r}(X ; G, A)=i m\left(i n f_{r}\right) \oplus k e r\left(d e f_{r}\right)$ where $\oplus$ denotes the direct sum of abelian groups. 
Proof. $\operatorname{def}_{r} \inf _{r}=h^{q}$ for some $q \geqq 1$ by Theorem 5.1. Since $h^{q}$ is an automorphism of $H^{r}(Y ; G, A)$, Theorem 7.1 follows from routine group arguments.

EXAMPLE 7.2. Consider the morphism $(G, G) \rightarrow(G, G / H)$ of Example 4.1 and suppose that, for some $r \in Z, H^{r}(G / H ; G, A)$ is uniquely divisible by $h$. Since $m H^{r}(G / H ; G, A)=0$ where $m=[G: H]$, it is

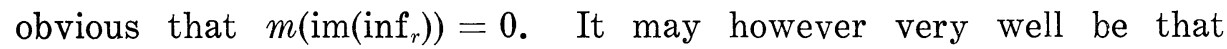
$H^{r}(X ; G, A)$, which is equal to $H^{r}(G, A)$, contains further elements which are annihilated by $m$. For instance, if $A$ is uniquely divisible by $h$, all elements of $H^{r}(X ; G, A)$ are annihilated by $m$. This follows from (1) $H^{r}(X ; G, A)$ is divisible by $h$ (it is even uniquely divisible by $h$ by Lemma 6.1$)$; (2) $m h H^{r}(X ; G, A)=0$ since $H^{r}(X ; G, A)=H^{r}(G$, $A$ ) and $m h$ is the order of $G$.

In this connection, it is interesting to recall that Faddeev proved in [4] that, if $H$ is a Hall subgroup of $G$, and $r \geqq 1$, im(inf ${ }_{r}$ ) consists of all the elements of $H^{r}(G, \mathrm{~A})$ which are annihilated by $m$. We conclude: Let $r \geqq 1$, let $A$ be uniquely divisible by $h$ and let $H$ be a Hall subgroup of $G$. Then, inf $f_{r}$ and def $f_{r}$ are both isomorphisms. In particular, $H^{r}(G, A) \simeq H^{r}(G: H, A)$. (This last isomorphism and the fact that inf is an isomorphism also follow from Faddeev's results on the restriction mapping. All one has to observe is that $H^{r}(H, A)=$ 0 , since $A$ is uniquely divisible by $h$.) The author conjectures that this result remains true for $r \leqq 0$.

8. The case that $A$ is uniquely divisible by $h$. We know from Lemma 6.1 that, if $A$ is uniquely divisible by $h$, Theorem 7.1 may be applied for all $r \in Z$. We now add to this that in this case $\inf _{r}=a_{r}^{*}$ and $\operatorname{def}_{r}=b_{r}^{*}$ for all $r \in Z$. In other words, the factor $h$ in Definitions 4.1 and 5.1 can be omitted. For deflation this is even correct if $h 1_{A}$ is only a monomorphism.

THEOREM 8.1. If $A$ is uniquely divisible by $h$, $\inf _{r}=a_{r}^{*}$ for all $r \in Z$. If $h 1_{A}$ is a monomorphism, def $f_{r}=b_{r}^{*}$ for all $r \in Z$.

Proof. Let $A$ be uniquely divisible by $h$ and select $r \in Z$. We see from diagram (III) that the $r$ th coboundary group of the lower row of that diagram is $B^{r}(Y ; G, A)$ if $r \geqq 1$ and is $h B^{r}(Y ; G, A)$ if $r \leqq 0$. We see from the proof of Lemma 6.1 that $B^{r}(Y ; G, A)$ is uniquely divisible by $h$ and hence $h B^{r}(Y ; G, A)=B^{r}(Y ; G, A)$. Since $\left\{a_{i}, i \in Z\right\}$ is a chain mapping it is now clear that $a_{r}\left(B^{r}(Y ; G, A)\right) \subset$ $B^{r}(X ; G, A)$; hence, by Definition 4.1, $\inf _{r}=a_{r}^{*}$.

Let $h 1_{A}$ be a monomorphism and select $r \in Z$. We see from 
diagram (IV) that the $r$ th cocycle group of the lower row of that diagram is $Z^{r}(Y ; G, A)$ if $r \leqq-2$ and is $\operatorname{ker}\left(h \delta_{r}^{\prime}\right)$ if $r \geqq-1$. Since $h 1_{A}$ is a monomorphism the endomorphism which consists of multiplying the elements of $C^{r+1}(Y ; G, A)$ by $h$ is evidently a mono; hence, $\operatorname{ker}\left(h \delta_{r}^{\prime}\right)=\operatorname{ker}\left(\delta_{r}^{\prime}\right)=Z^{r}(Y ; G, A)$. Since $\left\{b_{i}, i \in Z\right\}$ is a chain mapping it is now clear that $b_{r}\left(Z^{r}(X ; G, A)\right) \subset Z^{r}(Y ; G, A)$; hence, by Definition 5.1, def $_{r}=b_{r}^{*}$. Done.

We are now going to study inflation and deflation for dimensions $0,-1$, and 1 .

9. Inflation in dimension zero. We restrict ourselves in the remainder of this paper to the morphism $\left(1_{G}, f\right):(G, G) \rightarrow(G, G / H)$ of example 4.1. Hence, from now on, $X=G, Y=G / H, h=[H: 1]$ and $m=[G: H]$ where $m$ is the index of $(G, G / H)$. We denote the order of $G$ by $n$. The trace mapping $S_{G / H}: A^{B} \rightarrow A^{G}$ is the customary one; we usually write $S_{G}, S_{H}$ instead of $S_{G / 1}$ or $S_{H / 1}$.

We know that there exists an isomorphism $j: A \rightarrow C^{0}(X ; G, A)$ given by $(j(a))(1)=a$, where $a \in A$ and 1 is the unit element of $G$. (See Proposition 4.2 of [6].) The same reference tells us that there exists an isomorphism $k: A^{H} \rightarrow C^{0}(Y ; G, A)$ given by $(k(a))(H)=a$, where $a \in A^{H}$.

Proposition 9.1. The following diagram commutes

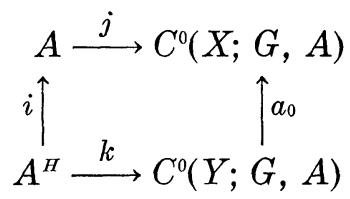

where $i: A^{H} \rightarrow A$ is the inclusion mapping.

Proof. Let $a \in A^{H}$. Then $(j i(a))(1)=i(a)=a$, while $\left(a_{0} k(a)\right)$ $(1)=(k(a))(H)=a$. Done.

We conclude that inflation for 0-cochains is nothing but the inclusion mapping $i$ : $A^{H} \rightarrow A$. Since $Z^{0}(Y ; G, A)=Z^{0}(X ; G, A)=A^{G}$ (see Proposition 4.1 of [6]) and $i \mid A^{G}$ is the identity, inflation for 0-cocycle is the identity mapping of $A^{\theta}$. We have observed in $\S 4$ that we cannot expect that $a_{0}\left(B^{0}(Y ; G, A)\right) \subset B^{0}(X ; G, A)$. Let's see what the situation is.

$B^{0}(Y ; G, A)=S_{G / H} A^{H}$ and $B^{0}(X: G, A)=S_{G} A$ by Proposition 4.3 of [6]. However the inclusion goes the wrong way, that is, $S_{G} A \subset$ $S_{G / H} A^{H}$ as follows from $S_{G} A=S_{G \mid H} S_{H} A \subset S_{G \mid H} A^{H}$. We conclude from Definition 4.1: 
Proposition 9.2. $\inf _{0}=a_{0}^{*}$ iff $S_{G / H} A^{H}=S_{G} A$. In that case, $\inf _{0}$ is the identity mapping of $A^{G} / S_{G} A$. Otherwise, $\inf _{0}\left(a+S_{G \mid H} A^{H}\right)=$ $h a+S_{G} A$ for all $a \in A^{G}$.

EXAMPLE 9.1 . Let $A=Z$ with trivial $G$-action. Then, $A^{H}=$ $A^{G}=Z, S_{g \mid H} A^{H}=m Z, S_{g} A-n Z$ and hence, if $H \neq\{1\}, S_{g^{\prime} H} A^{H} \neq S_{g} A$. Furthermore, $A^{G} / S_{G / H} A^{H}=Z_{m}$ (the cyclic group with $m$ elements) and $A^{G} / S_{G} A=Z_{n}$. We see from Proposition 9.2 that $\inf _{0}: Z_{m} \rightarrow Z_{n}$ is the natural monomorphism $z+m Z \rightarrow h z+n Z$ where $z \in Z$; this is also true if $H=\{1\}$. It is immediate from Proposition 9.2 that in general, if $G$ acts trivially on $A$ and $h 1_{A}$ is a monomorphism, inf is a monomorphism.

REMARK 9.1. We always have $h S_{G / H} A^{H} \subset S_{G} A \subset S_{G / H} A^{I}$. The right hand inclusion was observed before Proposition 9.2. The left hand inclusion follows either from $h a_{0}\left(B^{0}(Y ; G, A)\right) \subset B^{0}(X ; G, A)$ (see §4) or from $S_{G} A \supset S_{G} A^{H}=S_{G / H} S_{H} A^{H}=h S_{G \mid H} A^{H}$.

10. Deflation in dimension zero. Let $j: A \rightarrow C^{0}(X ; G, A)$ and $k: A^{H} \rightarrow C^{0}(Y ; G, A)$ denote the same isomorphism as in Proposition 9.1.

Proposition 10.1. The following diagram commutes.

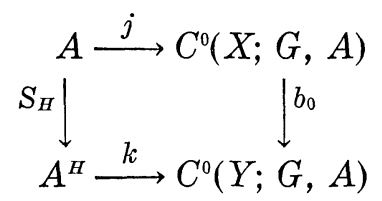

Proof. Let $a \in A$. Then, $\left(k S_{H} a\right)(H)=S_{H} a$, while $\left(b_{0} j(a)\right)(H)=$ $j(a)\left(\Sigma_{\rho \in H} \rho\right)=\Sigma_{\rho \varepsilon H} \rho a=S_{H} a$. Done.

We conclude that deflation for 0-cochains is the trace mapping $S_{H}: A \rightarrow A^{H}$. Furthermore, deflation for 0-cocycles consists of multiplying the elements of $A^{G}$ by $h$, since this is the effect of $S_{H}$ on $A^{G}$. This comes as a mild surprise since it shows that $b_{0}\left(Z^{0}(X ; G, A)\right) \subset$ $Z^{\circ}(Y ; G, A)$ which, as we observed in $\S 5$, can not be expected to be true for all morphisms of permutation representations. We know from the same section that $b_{0}\left(B^{\circ}(X ; G, A)\right) \subset B^{\circ}(Y ; G, A)$ which is equivalent to saying that $h S_{G} A \subset S_{G / H} A^{H}$; this last inclusion follows from $S_{G} A \subset S_{G \mid H} A^{H}$, observed before Proposition 9.2.

Since $S_{G} A \subset S_{G / H} A^{H} \subset A^{\theta}$, the natural epimorphism $\gamma: A^{\alpha} / S_{G} A \rightarrow A^{\alpha} /$ $S_{G / H} A^{H}$ is given by $\gamma\left(\alpha+S_{G} A\right)=a+S_{G / H} A^{H}$, where $a \in A$. It would have been nice if $\gamma$ had been $\operatorname{def}_{0}$, but we regretfully conclude from Definition 5.1:

Proposition 10.2. $\operatorname{def}_{0}=b_{0}^{*}$. Explicitly, $\operatorname{def}_{0}\left(a+S_{G} A\right)=h a+$ $S_{G \mid H} A^{H}$ for all $a \in A^{G}$; i.e., $\operatorname{def}_{0}=h \gamma$. 
ExAmple 10.1. Let $A=Z$ with trivial $G$-action. Then, $\operatorname{def}_{0}$ : $Z_{n} \rightarrow Z_{m}$ is $h \gamma$, where $\gamma: Z_{n} \rightarrow Z_{m}$ is the natural epimorphism given by $\gamma(z+n Z)=z+m Z$ for $z \in Z$. It is clear from this example that def may be neither a monomorphism nor an epimorphism.

11. Coboundaries in dimension -1 . In order to study inflation in dimension -1 we need some material on the $(-1)$-coboundaries of the permutation representation $(G, Y)=(G, G / H)$.

Let $\sigma_{1}, \cdots, \sigma_{m}$ be a set of representatives for the left cosets of $H$, i.e., $Y=G / H=\left\{\sigma_{1} H, \cdots, \sigma m H\right\}$. We assume that the enumeration is such that $\sigma_{1} H, \cdots, \sigma_{u} H(1 \leqq u \leqq m)$ is a set of representatives of the permutation representation $(H, G / H)$. (According to $\S 4$ of [6] this means that $(H, G / H)$ has $u$ domains of transitivity and that $\sigma_{i} H$ belongs to the $i$ th domain.) We shall use the following notation.

Notation 11.1. $H_{i}=H \cap \sigma_{i} H \sigma_{i}^{-1}$ and $M_{i}=H \cap \sigma_{i}^{-1} H \sigma_{i}$ for $i=1$, $\cdots, u$. Observe that $M_{i}=\sigma_{i}^{-1} H_{i} \sigma_{i}$.

Notation 11.2. $S_{i} \in Z[H]$ is the sum of a fixed set of representatives for the left cosets of $H_{i}$ as a subgroup of $H ; S_{i}^{\prime} \in Z[H]$ is the sum of a fixed set of representatives for the left cosets of $M_{i}$ as a subgroup of $H$, where $i=1, \cdots, u$. Hence the trace mapping $A^{H_{i}} \rightarrow$ $A^{H}\left(A^{M_{i}} \rightarrow A^{H}\right)$ consists of multiplying the elements of $A^{H_{i}}$ by $S_{i}$ (of $A^{M i}$ by $\left.S_{i}^{\prime}\right)$.

We must first get a hold on $C^{-2}(Y ; G, A)=\operatorname{Hom}_{G}\left(Z\left[Y^{2}\right], A\right)$.

Proposition 11.1. The permutation representation $\left(G, Y^{2}\right)$ has the pairs $\left(H, \sigma_{i} H\right)$ for $i=1, \cdots, u$ as a set of representatives.

Proof. Let $1 \leqq i \neq j \leqq u$. Then, $\sigma\left(H, \sigma_{i} H\right) \neq\left(H, \sigma_{j} H\right)$ for all $\sigma \in G$. Namely, $\sigma H=H$ means that $\sigma \in H$ and this implies that $\sigma \sigma_{i} H \neq \sigma_{j} H$. Now consider the arbitrary pair $(\sigma H, \tau H)$ of $Y^{2}$ where $\sigma, \tau \in G$. Then, $\sigma^{-1}(\sigma H, \tau H)=\left(H, \sigma^{-1} \tau H\right)$ and there exists a $\rho \in H$ such that $\rho \sigma^{-1} \tau H=\sigma_{i} H$ for some $1 \leqq i \leqq u$. Since $\rho \sigma^{-1}(\sigma H, \tau H)=$ $\left(H, \sigma_{i} H\right)$ we are done.

The subgroup of $G$ which leaves the pair $\left(H, \sigma_{i} H\right)$ fixed is the group $H_{i}$ of Notation $11.1 ; i, \cdots, u$. Hence we conclude from $\S 4$ of [6] that there exists an isomorphism $t: A^{H_{1}} \oplus \cdots \oplus A^{H_{u}} \rightarrow C^{-2}(Y ; G, A)$ given by: If $a_{i} \in A^{H_{i}}$ for $i=1, \cdots, u$, then $\left(t\left(a_{1}, \cdots, a_{u}\right)\right)\left(H, \sigma_{i} H\right)=$ $a_{i}$.

We can also consider the homomorphism $d_{-2}: A^{H_{1}} \oplus \cdots \oplus A^{H_{u}} \rightarrow$ $A^{H}$ given by $d_{-2}\left(a_{1}, \cdots, a_{u}\right)=\sum_{i=1}^{u}\left(S_{i}^{\prime}\left(\sigma_{i}^{-1} a_{i}\right)-S_{i} a_{i}\right)$ where again $a_{i} \in$ 
$A^{B_{i}}$ for $i=1, \cdots, u$. (It is immediate that, if $a_{i} \in A^{B_{i}}$, then $\sigma_{i}^{-1} a_{i} \in$ $A^{M_{i}}$.

Finally, since $C^{-1}(Y ; G, A)=C^{0}(Y ; G, A)$, there is available the isomorphism $k: A^{B} \rightarrow C^{-1}(Y ; G, A)$ of Proposition 9.1.

Proposition 11.2. The following diagram commutes.

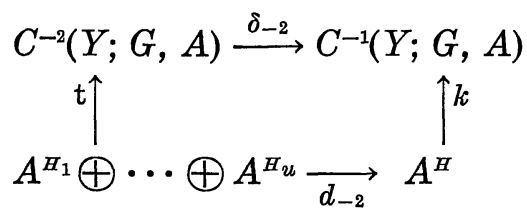

Proof. Let $a_{i} \in A^{H_{i}}$ for $i=1, \cdots, u$. Then $\left(k d_{-2}\left(a_{1}, \cdots, a_{u}\right)\right)$ $(H)=d_{-2}\left(a_{1}, \cdots, a_{u}\right)$. Furthermore, using the formula for $\delta_{-2}$ of $\S 1$ of [6], $\left(\delta_{-2} t\left(a_{1}, \cdots, a_{u}\right)\right)(H)=t\left(a_{1}, \cdots, a_{u}\right)\left(\sum_{j=1}^{m}\left(\sigma_{j} H, H\right)-\sum_{j=1}^{m}\left(H, \sigma_{j} H\right)\right)$. In order to compute the sum $\sum_{j=1}^{m}\left(H, \sigma_{j} H\right)$ we consider the permutation representation $\left(H,\left\{\left(H, \sigma_{1} H\right), \cdots,\left(H, \sigma_{m} H\right)\right\}\right)$. It is immediate that the pairs $\left(H, \sigma_{1} H\right), \cdots,\left(H, \sigma_{u} H\right)$ also form a set of representatives for this permutation representation. Since $H_{i}$ is the subgroup of $H$ which leaves $\left(H, \sigma_{i} H\right)$ fixed, $\sum_{j=1}^{m}\left(H, \sigma_{j} H\right)=\sum_{i=i}^{u} S_{i}\left(H, \sigma_{i} H\right)$ and hence $t\left(a_{1}\right.$, $\left.\cdots, a_{u}\right)\left(\sum_{j=1}^{m}\left(H, \sigma_{j} H\right)\right)=\sum_{i=1}^{u} S_{i} a_{i}$. In order to compute the sum $\sum_{j=1}^{m}\left(\sigma_{j} H, H\right)$ we consider the permutation representation $\left(H,\left\{\left(\sigma_{1} H, H\right)\right.\right.$, $\left.\left.\cdots,\left(\sigma_{m} H, H\right)\right\}\right)$. Since $\sigma_{i}^{-1}\left(H, \sigma_{i} H\right)=\left(\sigma_{i}^{-1} H, H\right)$ we see easily that the pairs $\left(\sigma_{i}^{-1} H, H\right), \cdots,\left(\sigma_{u}^{-1} H, H\right)$ from a set of representatives for this last permutation representation. Since $M_{i}$ is the subgroup of $H$ which leaves $\left(\sigma_{i}^{-1} H, H\right)$ fixed, $\sum_{j=1}^{m}\left(\sigma_{j} H, H\right)=\sum_{i=1}^{u} S_{i}^{\prime}\left(\sigma_{i}^{-1} H, H\right)$ and hence $t\left(a_{1}, \cdots, a_{u}\right)\left(\sum_{j=1}^{m}\left(\sigma_{j} H, H\right)\right)=\sum_{i=1}^{u} S_{i}^{\prime}\left(\sigma_{i}^{-1} a_{i}\right)$. We conclude that $\left(\delta_{-2}\right.$ $\left.t\left(a_{1}, \cdots, a_{u}\right)\right)(H)=\sum_{i=1}^{u}\left(S_{i}^{\prime}\left(\sigma_{i}^{-1} a_{i}\right)-S_{i} a_{i}\right)=d_{-2}\left(a_{1}, \cdots, a_{u}\right)$. Done.

REMARK 11.1. The above elements $\sigma_{1}, \cdots, \sigma_{u}$ are nothing but a set of representatives for the double cosets of $H$ as a subgroup of $G$. This remark makes it easy to check that our expression $\sum_{i=1}^{u}\left(S_{i}^{\prime}\right.$ $\left.\left(\sigma_{i}^{-1} a_{i}\right)-S_{i} a_{i}\right)$ for the $(-1)$-coboundaries of $(G, Y)$ is equivalent to, although not identical with, the expression $*$ on page 69 of [1].

We denote the kernel of the trace mapping $S_{G / H}: A^{H} \rightarrow A^{G}$ by $\operatorname{ker}\left(S_{\theta / H}\right)$. The ideal of $Z[G]$ which has as ideal base the elements $\sigma-1$, where $\sigma \in G$, is as usual denoted by $I$.

LEMMA 11.1. $\operatorname{im}\left(d_{-2}\right) \subset\left(I A \cap \operatorname{ker}\left(S_{G / H}\right)\right)$.

Proof. The following diagram commutes. 


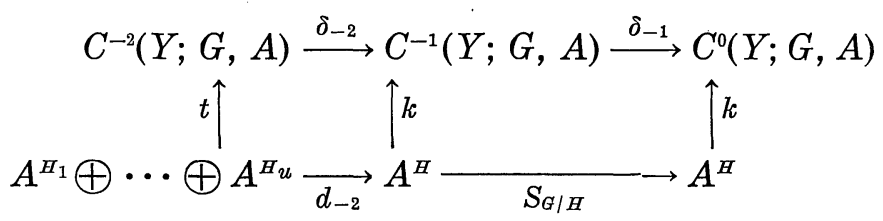

The left hand square commutes by Proposition 11.2; the right hand square commutes by $\S 4$ of [6]. Since $k$ is an isomorphism and $\delta_{-1} \delta_{-2}=$ 0 we read from this diagram that $S_{G / H} d_{-2}=0$, i.e., that $\operatorname{im}\left(d_{-2}\right) \subset$ $\operatorname{ker}\left(S_{G / H}\right)$. We now turn to $\operatorname{im}\left(d_{-2}\right) \subset I A$. We observe that the groups $H_{i}$ and $M_{i}$ of Notation 11.1 are conjugate (in $G$ ) and hence contain the same number, say $c_{i}$, of elements. Hence the two decompositions of $H$ into the left cosets of $H_{i}$, respectively $M_{i}$, both consist of subsets of $H$ with $c_{i}$ elements. We conclude from Theorem 4 on page 12 of [8] that there exists a common set of representatives for the left cosets of $H_{i}$ and of $M_{i}$ as subgroups of $H$. We now use such a common set of representatives to compute $S_{i}$ and $S_{i}^{\prime}$ of Notation 11.2, and obtain that $S_{i}=S_{i}^{\prime}$. Hence, if $a_{i} \in A^{H_{i}}$ for $i=1, \cdots, u, d_{-2}\left(a_{1}\right.$, $\left.\cdots, a_{u}\right)=\sum_{i=1}^{u} S_{i}\left(\sigma_{i}^{-1}-1\right) a_{i} \in I A$. Done.

CoRollary 11.1. If $G$ acts trivially on $A, \operatorname{im}\left(d_{-2}\right)=0$.

Proof. $G$ acts trivially on $A$ if and only if $I A=0$. Done.

12. Inflation in dimension -1 . The homomorphism $a_{-1}$ : $C^{-1}(Y ; G, A) \rightarrow C^{-1}(X ; G, A)$ is identical with the homomorphism $a_{0}$ : $C^{0}(Y ; G, A) \rightarrow C^{0}(X ; G, A)$. Consequently, Proposition 9.1 is valid with $a_{0}$ replaced by $a_{-1}$; i.e., $j i=a_{-1} k$. We conclude that inflation for $(-1)$-cochains is the inclusion mapping $i: A^{H} \rightarrow A$. Since $Z^{-1}(Y ; G$, $A)=\operatorname{ker}\left(S_{G / H}\right)$ and $Z^{-1}(X ; G, A)=\operatorname{ker}\left(S_{G}\right)$, inflation for $(-1)$-cocycles is the inclusion mapping $\operatorname{ker}\left(S_{G \mid H}\right) \rightarrow \operatorname{ker}\left(S_{G}\right)$. (The fact that $\operatorname{ker}\left(S_{G / H}\right) \subset \operatorname{ker}\left(S_{G}\right)$ follows from $\S 4$ or from $S_{G}=S_{G / H} S_{H}$.) Since $B^{-1}(Y ; G, A)=\operatorname{im}\left(d_{-2}\right)$ (see Proposition 11.2) and $B^{-1}(X ; G, A)=I A$ we see from Lemma 11.1 that $a_{-1}\left(B^{-1}(Y ; G, A)\right) \subset B^{-1}(X ; G, A)$; this could not have been predicted from $\S 4$. We conclude from Definition 4.1:

Proposition 12.1. $\inf _{-1}=a_{-1}^{*}$. Explicity, $\inf _{-1}\left(a+\operatorname{im}\left(d_{-2}\right)\right)=a+$ $I A$ for all $a \in \operatorname{ker}\left(S_{\theta / H}\right)$.

The following theorem is crucial for the duality theory of transitive permutation representations.

THEOREM 12.1. Let $d=(h, m)$. If $A$ is uniquely divisible by $d$, then $i m\left(d_{-2}\right)=I A \cap \operatorname{ker}\left(S_{G \mid H}\right)$. This happens for instance in each of the following two cases:

(a) $A$ is uniquely divisible by $h$;

(b) $H$ is a Hall subgroup of $G$. 
Proof. We see from Proposition 12.1 that $\operatorname{ker}\left(\inf _{-1}\right)=(I A \cap$ $\left.\operatorname{ker}\left(S_{G \mid H}\right)\right) / \operatorname{im}\left(d_{-2}\right)$. Lemma 7.1 and Theorem 7.1 tell us that $\inf _{-1}$ is a monomorphism if $A$ is uniquely divisible by $d$. The remainder of Theorem 12.1 follows from Proposition 7.1 and Example 7.1. Done.

13. Deflation in dimension -1 . The homomorphism $b_{-1}$ : $C^{-1}(X ; G, A) \cdots C^{-1}(Y ; G, A)$ is identical with the homomorphism $b_{0}$ : $C^{0}(X ; G, A) \rightarrow C^{\prime \prime}(Y ; G, A)$. Hence we conclude from Proposition 10.1 that deflation for (-1)-cochains is the trace mapping $S_{H}: A \rightarrow A^{H}$. It follows immediately from $S_{G}=S_{G / H} S_{H}$ that $S_{H}\left(\operatorname{ker}\left(S_{G)}\right) \subset \operatorname{ker}\left(S_{G / H}\right)\right.$, which signifies that $b_{\ldots 1}\left(Z^{-1}(X ; G, A)\right) \subset Z^{-1}(Y ; G, A)$; this could not have been predicted from $\S 5$. We conclude from Definition 5.1:

Proposition 13.1. $\operatorname{def}_{-1}=b_{-1}^{*}$. Explicitly, $\operatorname{def}_{-1}(a+I A)=S_{H} a+$ $\operatorname{im}\left(d_{-2}\right)$ for all $a \in \operatorname{ker}\left(S_{G}\right)$.

The following theorem is the dual of Theorem 12.1.

THEOREM 13.1. In each of the following two cases, $\operatorname{im}\left(d_{-2}\right)+$ $S_{H}\left(\operatorname{ker}\left(S_{G}\right)\right)=\operatorname{ker}\left(S_{G / H}\right)$.

(a) $A$ is uniquely divisible by $h$.

(b) $H$ is a Hall subgroup of $G$.

Proof. We see from Proposition 13.1 that $\operatorname{im}\left(\operatorname{def}_{-1}\right)=\left[\operatorname{im}\left(d_{-2}\right)+\right.$ $\left.S_{H}\left(\operatorname{ker}\left(S_{G}\right)\right)\right] / \operatorname{im}\left(\mathrm{d}_{-2}\right)$. Hence, $\operatorname{def}_{-1}$ is an epimorphism if and only if $\operatorname{im}\left(d_{-2}\right)+S_{H}\left(\operatorname{ker}\left(S_{G}\right)\right)=\operatorname{ker}\left(S_{G / H}\right)$. Proposition 7.1, Example 7.1 and Theorem 7.1 tell us that def $_{-1}$ is an epimorphism in each of the cases (a) and (b). Done.

Lemma 13.1. $S_{1 /}(I A) \subset \operatorname{im}\left(\mathrm{d}_{2}\right)$.

Proof. Since ${ }^{\prime}{ }^{\prime}(X ; G, A)=I A$ and $B^{-1}(Y ; G, A)=\operatorname{im}\left(d_{-2}\right)$, Lemma 13.1 is equivalent to saying that $b_{-1}\left(B^{-1}(X ; G, A)\right) \subset B^{-1}(Y ; G, A)$. This last inclusion was observed in $\S 5$. Done.

14. Inflation in climension 1 . We denote by $M$ the additive group of the crossed homomorphisms from $G$ to $A$; and by $M_{B}$ the subgroup of $M$ whose elements are zero on $H$. We know from $\S 6$ of [6] that there exists an isomorphism $v: Z^{1}(Y ; G, A) \rightarrow M_{H}$ which is defined by $(v c)(\sigma)=c(H, \sigma H)$ for $c \in Z^{1}(Y ; G, A)$ and $\sigma \in G$. Similarly, the isomorphism $w: Z^{\prime}(X ; G, A) \rightarrow M$ is defined by $(w c)(\sigma)=c(1, \sigma)$ where $c \in Z^{1}(X ; G, A), \sigma \in G$ and 1 is the unit element of $G$. We denote the inclusion mapping $M_{\|} \rightarrow M$ by $u$ and recall from $\S 4$ that $a_{1}\left(Z^{1}(Y ; G, A)\right) \subset Z^{1}(X ; G, A)$. 
Proposition 14.1. The following diagram commutes.

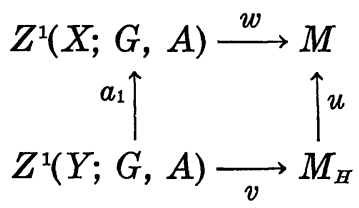

Proof. Let $c \in Z^{1}(Y ; G, A)$ and $\sigma \in G$. Then, $\left(\left(w a_{1}\right)(c)\right)(\sigma)=$ $\left(a_{1} c\right)(1, \sigma)=c(H, \sigma H)$; and $(u v(c))(\sigma)=(v c)(\sigma)=c(H, \sigma H)$. Done.

We conclude that inflation for 1-cocycles is the inclusion mapping $u: M_{H} \rightarrow M$. In order to study inflation for 1-coboundaries, we recall from $\S 6$ of [6] that $v\left(B^{-1}(Y ; G, A)\right)$ is the subgroup $M_{H}^{\prime}$ of $M_{H}$ which is described as follows: If $g \in M_{H}^{\prime}$ and $\sigma \in G$, then $g(\sigma)=(\sigma-1) a$ for some fixed $a \in A^{B}$. The subgroup $M^{\prime}=w\left(B^{1}(X ; G, A)\right)$ of $M$ is described similarly with $A^{B}$ replaced by $A$. Since $M_{H}^{\prime} \subset M^{\prime}$ we see that $a_{1}\left(B^{1}\right.$ $(Y ; G, A)) \subset B^{1}(X ; G, A)$ which checks with $\S 4$. We conclude from Definition 4.1:

Proposition 14.2. $\inf _{1}=a_{1}^{*}$. Explicitly, $\inf _{1}\left(g+M_{H}^{\prime}\right)=g+M^{\prime}$ for all $g \in M_{H}$.

It is well known that $\inf _{1}: H^{1}(Y ; G, A) \rightarrow H^{1}(X ; G, A)$ is always a monomorphism. (see Theorem 7.3 of [1] or Theorem 15.1 of [6].) This also follows from Proposition 14.2 and the observation that $M_{H}^{\prime}=$ $M^{\prime} \cap M_{H}$.

15. Endomorphisms of the group of crossed homomorphisms. Let $M$ and $M_{H}$ be as in the previous section. In order to study deflation in dimension 1 , we define what should be regarded as the natural homomorphism $D: M \rightarrow M_{H}$. If $g \in M$ and $\sigma \in G$ we denote the sum $\Sigma g(\gamma)$, where $\gamma$ runs through $\sigma H$, by $s_{g}(\sigma H)$. In particular $s_{g}(H)=$ $\Sigma g(\rho)$, where $\rho$ runs through $H$. We now define the homomorphism $D: M \rightarrow M_{H}$.

Definition 15.1. If $g \in M$ and $\sigma \in G,(D(g))(\sigma)=s_{g}(\sigma H)-s_{g}(H)$.

One proves routinely that $D$ is a homomorphism from $M$ into $M_{H}$. We observe that $s_{g}(\sigma H)=\Sigma g(\sigma \rho)$, where $\rho$ runs through $H$. Using that $g(\sigma \rho)=g(\sigma)+\sigma g(\rho)$, we find:

Proposition 15.1. If $g \in M$ and $\sigma \in G, \quad(D(g))(\sigma)=h g(\sigma)+$ $(\sigma-1) s_{g}(H)$.

ExAMPLE 15.1. Let $G$ act trivially on $A$. Then, $M=\operatorname{Hom}(G, A)$ and $M_{H}$ consists of those homomorphisms from $G$ to $A$ which vanish 
on $H$. If $g \in \operatorname{Hom}(G, A)$, we see from Proposition 15.1 that $D(g)=h g$ and indeed, multiplication by $h$ is the most naive way to change a homomorphism belonging to $\operatorname{Hom}(G, A)$ into one which is zero on $H$.

We now prepare for the study of $\operatorname{ker}(D)$.

\section{Proposition 15.2. If $g \in M, S_{H}\left(s_{0}(H)\right)=0$.}

Proof. Let $\rho \in H$. Then, $\rho s_{g}(H)=\Sigma \rho g(\gamma)$ where $\gamma$ runs through $H$. Since $g(\rho \gamma)=g(\rho)+\rho g(\gamma)$, this last sum equals $-h g(\rho)+s g(H)$. Consequently, $S_{H}\left(s_{\theta}(H)\right)=-h s_{\theta}(H)+h s_{\theta}(H)=0$. Done.

We know from $\S 6$ of [6] that the homomorphism $\delta_{0}^{\prime}: C^{0}(Y ; G, A) \rightarrow$ $Z^{1}(Y ; G, A)$ may be interpreted as the homomorphism $\delta_{0}^{\prime}: A^{B} \rightarrow M_{H}$, where $\left(\delta_{0}^{\prime}(a)\right)(\sigma)=(\sigma-1) a$ for $a \in A^{B}$ and $\sigma \in G$. Similarly, the homomorphism $\delta_{0}: C^{0}(X ; G, A) \rightarrow Z^{1}(X ; G, A)$ may be interpreted as the homomorphism $\delta_{0}: A \rightarrow M$, where $\left(\delta_{0}(a)\right)(\sigma)=(\sigma-1) a$ for $a \in A$ and $\sigma \in G$. We also recall from $\S 10$ that the homomorphism $b_{0}: C^{0}(X ; G, A) \rightarrow C^{0}(Y ; G, A)$ may be interpreted as the homomorphism $S_{H}: A \rightarrow A^{H}$.

Proposition 15.3. The following diagram commutes.

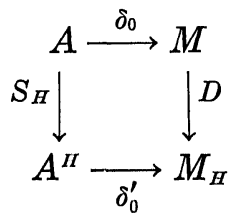

Proof. Let $a \in A$ and $\sigma \in G$. Then $\left(\delta_{0}^{\prime} S_{H}(a)\right)(\sigma)=(\sigma-1) S_{H}(a)$. Furthermore, denoting $\delta_{0}(a)=g,\left(D \delta_{0}(a)\right)(\sigma)=(D g)(\sigma)=h g(\sigma)+(\sigma-$ 1) $s_{g}(H)=h(\sigma-1) a+(\sigma-1) \Sigma(\rho-1) a$ where $\rho$ runs through $H$. Since $\Sigma(\rho-1) a=S_{u}(a)-h a,\left(D \delta_{0}(a)\right)(\sigma)=(\sigma-1) S_{H}(a)$. Done.

If $K$ is a subgroup of $M$ we denote the larger subgroup $\{g \mid g \in M$, $h g \in K\}$ by $K: h$. We continue the investigation of the diagram of Proposition 15.3.

Proposition 15.4. $\quad h \operatorname{ker}(D) \subset \delta_{0}\left(\operatorname{ker}\left(S_{H}\right)\right) \subset \operatorname{ker}(D)$. If $h 1_{A}$ is a monomorphism, $\operatorname{ker}(D)=\delta_{0}\left(\operatorname{ker}\left(S_{H}\right)\right): h$.

Proof. The inclusion $\delta_{0}\left(\operatorname{ker}\left(S_{H}\right)\right) \subset \operatorname{ker}(D)$ is read immediately from the commutative diagram of Proposition 15.3. In order to show that $\left.h \operatorname{ker}(D) \subset \delta_{0}(\operatorname{ker}) S_{H}\right)$ ), we select $g \in \operatorname{ker}(D)$ and show that $h g \in$ $\delta_{0}\left(\operatorname{ker}\left(S_{H}\right)\right)$. That is, we prove that for all $\sigma \in G, h g(\sigma)=(\sigma-1) a$ for some fixed $a \in \operatorname{ker}\left(S_{H}\right)$. We see from Proposition 15.1 that $h g(\sigma)=$ $(\sigma-1)\left(-s_{g}(H)\right)$ and from Proposition 15.2 that $-s_{g}(H) \in \operatorname{ker}\left(S_{H}\right)$. 
The first line of Proposition 15.4 has now been proved. We conclude from it that $\operatorname{ker}(D) \subset \delta_{0}\left(\operatorname{ker}\left(S_{H}\right)\right): h \subset \operatorname{ker}(D): h$. If $h 1_{A}$ is a monomorphism, $h 1_{M}$ is a monomorphism and hence $\operatorname{ker}(D)=\operatorname{ker}(D): h$. Done.

REMARK 15.1. We shall see in the next section that the homomorphism $h D: M \rightarrow M_{H}$ is precisely the deflation for 1-cocycles. Clearly, $\operatorname{ker}(h D)=\operatorname{ker}(D): h$ and hence we have good information about the kernel of the deflation mapping.

16. Deflation in dimension 1. One proves easily that the isomorphism $v: Z^{1}(Y ; G, A) \rightarrow M_{H}$ of Proposition 14.1 has as inverse the isomorphism $v^{\prime}: M_{H} \rightarrow Z^{1}(Y ; G, A)$ defined by: If $g \in M_{H}$ and $\sigma$, $\tau \in G$, then $\left(v^{\prime}(g)\right)(\sigma H, \tau H)=g(\tau)-g(\sigma)$. (The proof uses that $g \in M_{H}$ if and only if $g \in M$ and $g$ is constant on the left cosets of $H$.) We shall regard $v^{\prime}$ as a monomorphism $v^{\prime}: M_{H} \rightarrow C^{1}(Y ; G, A)$. Similarly, we have the monomorphism $w^{\prime}: M \rightarrow C^{1}(X ; G, A)$ defined by: If $g \in M$ and $\sigma, \tau \in G$, then $\left(w^{\prime}(g)\right)(\sigma, \tau)=g(\tau)-g(\sigma)$.

Proposition 16.1. The following diagram commutes.

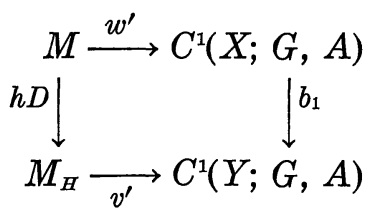

Proof. Let $g \in M$ and $\sigma, \tau \in G$. Then, using Definition 15.1, $\left(v^{\prime} h D(g)\right)(\sigma H, \tau H)=(h D(g))(\tau)-(h D(g))\left((\sigma)=h\left(s_{g}(\tau H)-s_{g}(\sigma H)\right)\right.$. Furthermore $\left(b_{1} w^{\prime}(g)\right)(\sigma H, \tau H)=w^{\prime}(g)(\Sigma(\sigma \rho, \tau \gamma))$, where the summation is over all pairs $(\rho, \gamma) \in H \times H$. Consequently, $\left(b_{1} w^{\prime}(g)\right)(\sigma H, \tau H)=$ $\Sigma(g(\tau \gamma)-g(\sigma \rho))=h s_{g}(\tau H)-h s_{g}(\sigma H)$. Done.

We conclude that deflation for 1-cocycles is the mapping $h D$ : $M \rightarrow M_{H}$. We see that $b_{1}\left(Z^{1}(X: G, A)\right) \subset Z^{1}(Y ; G, A)$ which could not have been predicted from $\S 5$. In order to study deflation for 1coboundaries we return to the groups $M^{\prime}$ and $M_{H}^{\prime}$ of $\S 14$.

\section{Proposition 16.2. $D\left(M^{\prime}\right) \subset M_{H}^{\prime}$ •}

Proof. We read from the diagram of Proposition 15.3 that $D \delta_{0}(A)=\delta_{0}^{\prime} S_{H}(A)$. Since $\delta_{0}(A)=M^{\prime}$ and $\delta_{0}^{\prime} S_{H}(A) \subset \delta_{0}^{\prime}\left(A^{H}\right)=M_{H}^{\prime}$, we are done.

It follows trivially from Proposition 16.2 that $h D\left(M^{\prime}\right) \subset M_{H}^{\prime}$, i.e., that $b_{1}\left(B^{1}(X ; G, A)\right) \subset B^{1}(Y ; G, A)$ which checks with $\S 5$. We conclude from Definition 5.1: 
Proposition 16.3. def $\quad b_{1}^{*}$. Explicitly, $\operatorname{def}_{1}\left(g+M^{\prime}\right)=h D(g)+$ $M_{H}^{\prime}$ for all $g \in M$.

Remark 16.1. Proposition 16.2 shows that $D$ induces a homomorphism $D^{*}: H^{1}(X ; G, A) \cdots H^{\prime}(Y ; G, A)$, given by $D^{*}\left(g+M^{\prime}\right)=D(g)+$ $M_{H}^{\prime}$ for all $g \in M$. Evidently, $D^{*}$ is the natural mapping from $H^{1}(X$; $G, A)$ into $H^{1}(Y ; G, A)$ and $\operatorname{def}_{1}=h D^{*}$. The factor $h$ is pure waste; and that, in times of deflation!

\section{REFERENCES}

1. I. T. Adamson, Cohomology Theory for Non-Normal Subgroups and Non-Normal Fields, Proc. Glascow Math., Assoc. 2(1954), 66-76.

2. W. Burnside, Theory of Groups of Finite Order, Dover Publications, second edition (1955).

3. H. Cartan and S. Eilenberg, Homological Algebra, Princeton University Press (1956)

4. D. K. Faddeev, On Homology Theory For Finite Groups of Operators, Izv. Akad. Nauk SSSR. Ser. Mat., 19(1955), 193-200 (Russian).

5. A. Grothendieck, Sur Quelques Points $D^{\prime}$ Algébre Homologique, Tôhoku Math. J., 9 (1957), 119-221.

6. E. Snapper, Cohomology of Permutation Representations. I. Spectral Sequences, J. Of Math. and Mech., 13(1964), 133-161.

7. E. Weiss, A Deflation Map, J. of Math. and Mech., 8(1959), 309-329.

8. H. Zassenhaus, The Theory of Groups, Chelsea Publishing Company, (1949).

Dartmouth College 



\section{PACIFIC JOURNAL OF MATHEMATICS}

\section{EDITORS}

\author{
H. Samelson \\ Stanford University \\ Stanford, California \\ R. M. Blumenthal \\ University of Washington \\ Seattle, Washington 98105
}

\author{
J. Dugundu \\ University of Southern California \\ Los Angeles, California 90007 \\ *Richard Arens \\ University of California \\ Los Angeles, California 90024
}

\section{ASSOCIATE EDITORS}
E. F. BeCKENBACH
B. H. NeUMANN
F. WOLF
K. YosidA

\section{SUPPORTING INSTITUTIONS}

\author{
UNIVERSITY OF BRITISH COLUMBIA \\ CALIFORNIA INSTITUTE OF TECHNOLOGY \\ UNIVERSITY OF CALIFORNIA \\ MONTANA STATE UNIVERSITY \\ UNIVERSITY OF NEVADA \\ NEW MEXICO STATE UNIVERSITY \\ OREGON STATE UNIVERSITY \\ UNIVERSITY OF OREGON \\ OSAKA UNIVERSITY \\ UNIVERSITY OF SOUTHERN CALIFORNIA
}

\author{
STANFORD UNIVERSITY \\ UNIVERSITY OF TOKYO \\ UNIVERSITY OF UTAH \\ WASHINGTON STATE UNIVERSITY \\ UNIVERSITY OF WASHINGTON \\ * * * * \\ AMERICAN MATHEMATICAL SOCIETY \\ CALIFORNIA RESEARCH CORPORATION \\ SPACE TECHNOLOGY LABORATORIES \\ NAVAL ORDNANCE TEST STATION
}

Mathematical papers intended for publication in the Pacific Journal of Mathematics should by typewritten (double spaced). The first paragraph or two must be capable of being used separately as a synopsis of the entire paper. It should not contain references to the bibliography. No separate author's resumé is required. Manuscripts may be sent to any one of the four editors. All other communications to the editors should be addressed to the managing editor, Richard Arens, at the University of California, Los Angeles, California 90024.

50 reprints per author of each article are furnished free of charge; additional copies may be obtained at cost in multiples of 50 .

The Pacific Journal of Mathematics is published quarterly, in March, June, September, and December. Effective with Volume 13 the price per volume (4 numbers) is $\$ 18.00$; single issues, $\$ 5.00$. Special price for current issues to individual faculty members of supporting institutions and to individual members of the American Mathematical Society: $\$ 8.00$ per volume; single issues $\$ 2.50$. Back numbers are available.

Subscriptions, orders for back numbers, and changes of address should be sent to Pacific Journal of Mathematics, 103 Highland Boulevard, Berkeley 8, California.

Printed at Kokusai Bunken Insatsusha (International Academic Printing Co., Ltd.), No. 6, 2-chome, Fujimi-cho, Chiyoda-ku, Tokyo, Japan.

PUBLISHED BY PACIFIC JOURNAL OF MATHEMATICS, A NON-PROFIT CORPORATION

The Supporting Institutions listed above contribute to the cost of publication of this Journal, but they are not owners or publishers and have no responsibility for its content or policies.

* Basil Gordon, Acting Managing Editor until February 1, 1966. 


\section{Pacific Journal of Mathematics}

\section{Vol. 15, No. $3 \quad$ November, 1965}

David R. Arterburn and Robert James Whitley, Projections in the space of

bounded linear operators .................................

Robert McCallum Blumenthal, Joram Lindenstrauss and Robert Ralph Phelps,

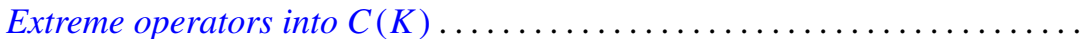

L. Carlitz, A note on multiple exponential sums ................... 757

Joseph A. Cima, A nonnormal Blaschke-quotient .................... 767

Paul Civin and Bertram Yood, Lie and Jordan structures in Banach algebras . . . 775

Luther Elic Claborn, Dedekind domains: Overrings and semi-prime

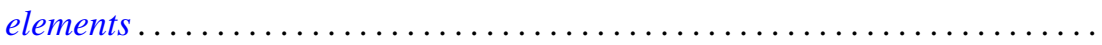

799

Luther Elic Claborn, Note generalizing a result of Samuel's .............. 805

George Bernard Dantzig, E. Eisenberg and Richard Warren Cottle, Symmetric

dual nonlinear programs ................................... 809

Philip J. Davis, Simple quadratures in the complex plane ............... 813

Edward Richard Fadell, On a coincidence theorem of F. B. Fuller ............ 825

Delbert Ray Fulkerson and Oliver Gross, Incidence matrices and interval

graphs ........................................ 835

Larry Charles Grove, Tensor products over $H^{*}$-algebras ..................

Deborah Tepper Haimo, $L^{2}$ expansions in terms of generalized heat polynomials

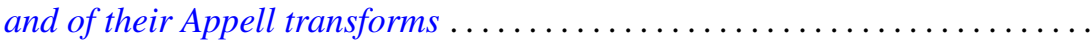

I. Martin (Irving) Isaacs and Donald Steven Passman, A chardcterization of groups in terms of the degrees of their characters ..........

Donald Gordon James, Integral invariants for vectors over local fields........ 905

Fred Krakowski, A remark on the lemma of Gauss ................... 917

Marvin David Marcus and H. Minc, A subdeterminant inequality ........... 921

Kevin Mor McCrimmon, Norms and noncommutative Jordan algebras ........ 925

Donald Earl Myers, Topologies for Laplace transform spaces ............... 957

Olav Njstad, On some classes of nearly open sets .................... 961

Milton Philip Olson, A characterization of conditional probability ........... 971

Barbara Osofsky, A counter-example to a lemma of Skornjakov .............. 985

Sidney Charles Port, Ratio limit theorems for Markov chains ............... 989

George A. Reid, A generalisation of $W^{*}$-algebras ...................... 1019

Robert Wells Ritchie, Classes of recursive functions based on Ackermann's

function ........................................... 1027

Thomas Lawrence Sherman, Properties of solutions of $n$th order linear

differential equations ........................................ 1045

Ernst Snapper, Inflation and deflation for all dimensions . .............. 1061

Kondagunta Sundaresan, On the strict and uniform convexity of certain Banach

spaces............................................. 1083

Frank J. Wagner, Maximal convex filters in a locally convex space .......... 1087

Joseph Albert Wolf, Translation-invariant function algebras on compact 